\title{
Efficacy and Safety of Anlotinib in Advanced Non-Small Cell Lung Cancer: A Real-World Study
}

This article was published in the following Dove Press journal: Cancer Management and Research

\section{Kun Zhang ${ }^{1,2}$ \\ Xiya $\mathrm{Ma}^{2}$ \\ Hongjun $\mathrm{Gao}^{2}$ \\ Hong Wang ${ }^{2}$ \\ Haifeng Qin (iD) ${ }^{2}$ \\ Shaoxing Yang ${ }^{2}$ \\ Xiaoqing Liu $^{2}$}

'Academy of Military Medical Science, Beijing 100089, People's Republic of China; ${ }^{2}$ Department of Lung Oncology,

The Fifth Medical Center of Chinese PLA General Hospital, Beijing 10007I, People's Republic of China
Correspondence: Xiaoqing Liu Department of Lung Oncology, The Fifth Medical Center of Chinese PLA General Hospital, No. 8 East Street, Fengtai District, Beijing 10007I, People's Republic of China

Tel + 8610-66947963

$\mathrm{Fax}+8610-51128605$

Email liuxiaoqing06I2@I63.com
Purpose: The ALTER0303 trial showed that anlotinib, a novel antiangiogenic tyrosine kinase inhibitor, administered as third-line or further treatment prolonged progression-free survival (PFS) and overall survival (OS) in patients with advanced non-small cell lung cancer (NSCLC). This retrospective study investigated the efficacy and safety of anlotinib in real-world settings.

Patients and Methods: Medical records of patients with advanced NSCLC receiving anlotinib as third-line or further treatment were collected, and survival curves were derived using the Kaplan-Meier method. Univariate analysis was performed by log-rank testing. Cox regression analysis was used to evaluate the significance of factors obtained from the univariate analysis.

Results: Fifty-two patients with advanced NSCLC were included. The objective response rate was $16 \%$, and the disease control rate was $80 \%$. The median PFS was 4.5 months $(95 \%$ confidence interval [CI]: 3.6-5.4), and the median OS was 9 months (95\% CI: $6.5-11.5$ ). Univariate analysis revealed that the group of patients with longer PFS and OS included Eastern Cooperative Oncology Group performance status (ECOG PS) $\leq 1, \leq 2$ distant metastases, no liver metastases, $\leq 3$ previous treatment lines, and $\leq 2$ previous chemotherapy lines. Cox regression analysis demonstrated that only patients with ECOG PS $\leq 1$ or no liver metastases had longer PFS and OS. Grade 3 treatment-related adverse events were reported in $14 \%$ of the patients, but no life-threatening adverse events were reported.

Conclusion: Anlotinib was well tolerated and effective in patients with advanced NSCLC in real-world conditions. Patients with ECOG PS $\leq 1$ or no liver metastases have longer PFS and OS.

Keywords: anlotinib, non-small cell lung cancer, antiangiogenesis, third-line or further treatment, real-world study

\section{Introduction}

Globally, lung cancer is associated with the highest morbidity and mortality among all cancers, and most patients already have advanced or metastatic disease when diagnosed. ${ }^{1}$ Non-small cell lung cancer (NSCLC) accounts for $80-85 \%$ of lung cancers. ${ }^{2}$ Therapeutic options for advanced NSCLC include chemotherapy, targeted therapy, immunotherapy, and antiangiogenic therapy. ${ }^{3}$ Angiogenesis plays an important role in tumor growth and metastasis, and vascular endothelial growth factor (VEGF) and its receptor (VEGFR) are involved in important signaling pathways associated with tumor angiogenesis. Thus, VEGF/VEGFR2 monoclonal antibodies and tyrosine kinase inhibitors (TKI) targeting VEGFR2 are common antiangiogenic drugs used in cancer treatment. ${ }^{4}$ 
Anlotinib, a novel TKI that targets VEGFR, plateletderived growth factor receptor, and fibroblast growth factor receptor, has significant antiangiogenic and antitumor activity. ${ }^{5}$ In a Phase III clinical trial (ALTER0303), anlotinib prolonged the median progression-free survival (PFS) by 4 months and the median overall survival (mOS) by 3.3 months in patients with advanced NSCLC compared with the placebo. ${ }^{6}$ Anlotinib was subsequently approved by the National Medical Products Administration of China for third-line or further treatment in patients with advanced NSCLC. More than $90 \%$ of patients with cancer are treated outside randomized clinical trials, and therefore, a real-world study presents an important way to obtain patient data that cannot be obtained from randomized clinical trials and to monitor post-marketing safety and adverse events. ${ }^{7}$ In this study, we analyzed the efficacy and safety of anlotinib in patients with advanced NSCLC in real-world settings.

\section{Patients and Methods \\ Data Source and Study Design}

This study was approved by the Ethics Committee of the Fifth Medical Center of the General Hospital of Chinese People's Liberation Army and was conducted according to the principles of the Declaration of Helsinki. As this retrospective study did not harm the rights and health of patients, and protected their privacy and personal information, the ethics committee waived the requirement to obtain informed consent. Eligible patients included those with advanced NSCLC (stage III B or IV) confirmed by histological and cytological examination, who were administered anlotinib as third-line or further treatment at the Department of Lung Oncology of the Fifth Medical Center of the General Hospital of the Chinese People's Liberation Army between June 1, 2018 and Dec 31, 2018. Patients with brain metastases were also included. Anlotinib was administered if there was disease progression after at least one line of chemotherapy and TKI therapy for all patients with driver alterations and after at least two lines of chemotherapy for patients without driver alterations. Gene expression status was determined using amplification-refractory mutation system, fluorescence in situ hybridization, or next-generation sequencing.

All patients received anlotinib $(12 \mathrm{mg} /$ day, 21-day cycles, defined as 2 weeks on treatment and 1 week off treatment) until disease progression, intolerable toxicity, or death. Dose modifications of anlotinib (10 or $8 \mathrm{mg} /$ day) were allowed. If anlotinib was not tolerated at $8 \mathrm{mg} / \mathrm{day}$, treatment was discontinued.

The efficacy of anlotinib was assessed using the Response Evaluation Criteria in Solid Tumors version 1.1 (RECIST 1.1) using computed tomography or nuclear magnetic resonance imaging. Progression-free survival (PFS) was defined as the time from the beginning of anlotinib treatment to tumor progression or patient death. Overall survival (OS) was defined as the time from the beginning of anlotinib treatment to patient death. The data cutoff was November 20, 2019. Adverse events were captured according to the Common Terminology Criteria for Adverse Events version 4.0.

\section{Statistical Analyses}

All statistical analyses were performed using SPSS 22.0. Survival analyses were performed using the Kaplan-Meier method, and the log-rank method was used for univariate analysis of PFS and OS. Cox regression was used to estimate significantly different factors in univariate analysis. Statistical significance was defined as $P<0.05$.

\section{Results}

\section{Patient Characteristics}

Fifty-two patients with advanced NSCLC who received anlotinib as third- or later-line treatment from Jun 1 to Dec 31, 2018 were recruited; of these, $24(46 \%)$ were female, 20 (38\%) aged $\geq 65$ years, $21(40 \%)$ had a smoking history, 10 $(19 \%)$ had an ECOG PS of 2, and 26 (50\%) harbored EGFR mutation; however, no other driver mutation was detected. Other clinical characteristics of the patients, such as clinical stage and pathological type, are shown in Table 1.

\section{Clinical Efficacy}

Two patients discontinued anlotinib treatment during the first cycle owing to grade 3 hypertension or hemoptysis caused by anlotinib. The best overall responses as per RECIST 1.1 among the remaining 50 patients were as follows: partial response (PR) in 8 patients, stable disease (SD) in 32 patients, and progressive disease (PD) in 10 patients. The objective response rate (ORR) was $16 \%$, and the disease control rate (DCR) was $80 \%$.

At the time of data cutoff, 47 (94\%) patients showed disease progression. The mPFS was 4.5 months $(95 \% \mathrm{CI}$ : 3.6-5.4; Figure 1A). Univariate analysis showed that PFS was significantly prolonged in cases of ECOG PS $\leq 1, \leq 2$ distant metastases, no liver metastases, $\leq 3$ previous 
Table I Baseline Characteristics of Patients

\begin{tabular}{|c|c|}
\hline Characteristic & Patients $(n=52)$ \\
\hline \multicolumn{2}{|l|}{ Sex } \\
\hline Male & $28(54 \%)$ \\
\hline Female & $24(46 \%)$ \\
\hline \multicolumn{2}{|l|}{ Age } \\
\hline$<65$ & $32(62 \%)$ \\
\hline $65-75$ & $10(19 \%)$ \\
\hline$\geq 75$ & $10(19 \%)$ \\
\hline \multicolumn{2}{|l|}{ Smoking history } \\
\hline Yes & 21 (40\%) \\
\hline No & $31(60 \%)$ \\
\hline \multicolumn{2}{|l|}{ ECOG PS } \\
\hline$\leq 1$ & $42(81 \%)$ \\
\hline 2 & $10(19 \%)$ \\
\hline \multicolumn{2}{|l|}{ Pathological type } \\
\hline Adenocarcinoma & $38(73 \%)$ \\
\hline Squamous cell carcinoma & $14(27 \%)$ \\
\hline \multicolumn{2}{|l|}{ Gene status } \\
\hline EGFR mutation & $26(50 \%)$ \\
\hline Wide type/unknown & $26(50 \%)$ \\
\hline \multicolumn{2}{|l|}{ Clinical stage } \\
\hline III B & $10(19 \%)$ \\
\hline IV & $42(81 \%)$ \\
\hline \multicolumn{2}{|l|}{ Number of distant metastases } \\
\hline$\leq 2$ & $38(73 \%)$ \\
\hline$>2$ & 14 (27\%) \\
\hline \multicolumn{2}{|l|}{ Brain metastases } \\
\hline Yes & $18(35 \%)$ \\
\hline No & $34(65 \%)$ \\
\hline \multicolumn{2}{|l|}{ Liver metastases } \\
\hline Yes & $8(15 \%)$ \\
\hline No & $44(85 \%)$ \\
\hline \multicolumn{2}{|l|}{ Number of previous treatment lines } \\
\hline$\leq 3$ & 42 (8I\%) \\
\hline$>3$ & $10(19 \%)$ \\
\hline \multicolumn{2}{|l|}{ Number of previous chemotherapy lines } \\
\hline$\leq 2$ & $40(77 \%)$ \\
\hline$>2$ & $12(23 \%)$ \\
\hline \multicolumn{2}{|l|}{ Previous EGFR-TKI treatment } \\
\hline Yes & $29(56 \%)$ \\
\hline No & $23(44 \%)$ \\
\hline \multicolumn{2}{|l|}{ Previous antiangiogenic treatment } \\
\hline Yes & $25(48 \%)$ \\
\hline No & $27(52 \%)$ \\
\hline
\end{tabular}

Abbreviations: ECOG PS, Eastern Cooperative Oncology Group performance status; EGFR, endothelial growth factor receptor; TKI, tyrosine kinase inhibitor. treatments lines, and $\leq 2$ previous chemotherapy lines (Figure 1B-F). Sex, age, smoking history, clinical stage, pathology, EGFR status, brain metastases, previous EGFR-TKI treatment, and previous antiangiogenic treatments had no influence on PFS (Table 2). Cox regression analysis indicated that only patients with ECOG PS $\leq 1$ (hazard ratio [HR]: $0.308,95 \%$ CI: $0.141-0.673$ ) or no liver metastases (HR: $0.197,95 \%$ CI: $0.079-0.489$ ) had a longer PFS (Table 3).

At the time of data cutoff, $38(76 \%)$ patients died. The mOS was 9 months (95\% CI: 6.5-11.5; Figure 2A). Univariate analysis showed that OS was significantly prolonged in cases of ECOG PS $\leq 1, \leq 2$ distant metastases, no liver metastases, $\leq 3$ previous treatments lines, and $\leq 2$ previous chemotherapy lines (Figure 2B-F). Sex, age, smoking history, clinical stage, pathology, EGFR status, brain metastases, previous EGFR-TKI treatment, and previous antiangiogenic treatments had no influence on OS (Table 4). Cox regression analysis indicated that only patients with ECOG PS $\leq 1$ (HR: $0.302,95 \%$ CI: $0.091--$ 0.684 ) or no liver metastases (HR: $0.065,95 \%$ CI: $0.004-$ 0.214 ) had a longer OS (Table 5).

\section{Safety}

Among the 52 patients receiving anlotinib, all treatmentrelated adverse events (Grade 1-3) were reported in 47 patients $(90.4 \%)$, including hypertension $(25 \%)$, fatigue (21\%), hand-foot syndrome (21\%), proteinuria $(17 \%)$, anorexia (15\%), constipation (13\%), diarrhea $(13 \%)$, oral mucositis (12\%), abnormal liver function (12\%), cough $(10 \%)$, hemoptysis $(10 \%)$, rash $(8 \%)$, and thrombocytopenia (6\%). Grade 3 treatment-related adverse events were reported in $14 \%$ of the patients, including hypertension $(8 \%)$, fatigue $(2 \%)$, proteinuria $(2 \%)$, cough $(2 \%)$, and hemoptysis (2\%). No life-threatening adverse events were reported (Table 6).

Among the 50 patients who received anlotinib over more than one cycle, dose reductions were required in five patients (10\%); of these, four patients received $10 \mathrm{mg} /$ day and one patient received $8 \mathrm{mg} /$ day. There were no dose discontinuations.

\section{Discussion}

This retrospective study evaluated the efficacy and safety of anlotinib, a novel antiangiogenic drug, as a third- or further-line treatment against advanced NSCLC. The 

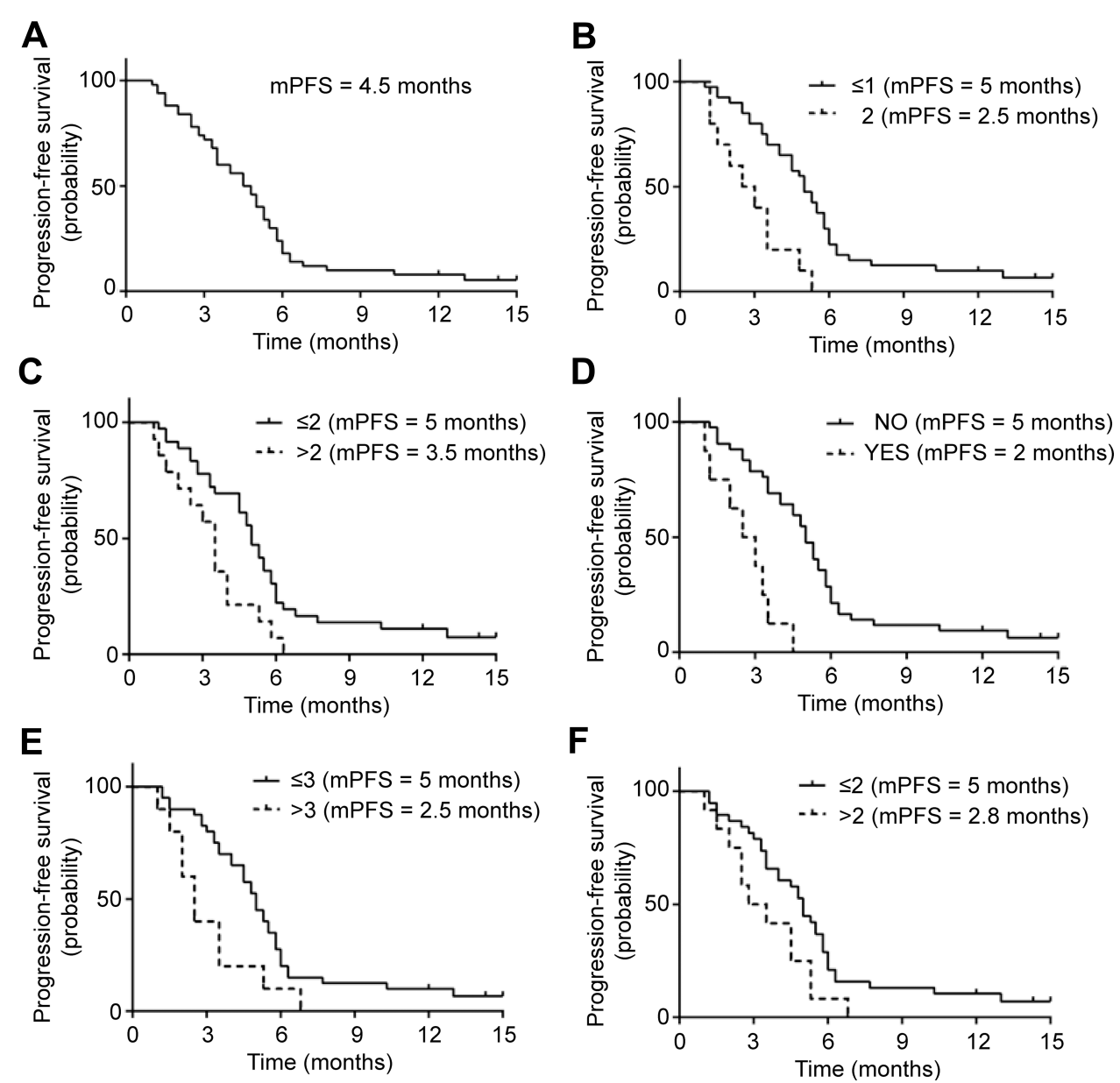

Figure I Progression-free survival of patients with advanced non-small cell lung cancer treated with anlotinib. (A) total population ( $\mathrm{n}=50$ ), (B) Eastern Cooperative Oncology Group performance status (ECOG PS), (C) number of distant metastases, (D) liver metastases, (E) number of previous treatment lines, (F) number of previous chemotherapy lines.

results indicated that anlotinib is effective and well tolerated in advanced NSCLC.

Antiangiogenic drugs have shown curative effects when used as first- and second-line treatments against advanced NSCLC. ${ }^{3}$ Bevacizumab in combination with chemotherapy has been demonstrated to improve PFS and OS compared with chemotherapy alone as first-line treatment in patients with advanced NSCLC. ${ }^{7-9}$ Likewise, ramucirumab combined with docetaxel has been shown to improve PFS and OS in patients with advanced NSCLC exhibiting resistance to platinum-based chemotherapy compared with docetaxel treatment alone, ${ }^{10}$ and ramucirumab/bevacizumab combined with erlotinib could improve PFS compared with erlotinib treatment alone as the first-line treatment in advanced patients with EGFRmutant NSCLC. ${ }^{11,12}$ However, most VEGFR TKIs can only prolong PFS, and not OS, when used as third- or later-line treatment in advanced NSCLC. ${ }^{13}$ A randomized phase III clinical trial comparing anlotinib with placebo as third- or later-line treatment for patients with NSCLC reported an increase in ORR (9.2 vs $0.7 \%, \mathrm{P}<0.001)$ and DCR (81.0 vs $37.1 \%, \mathrm{P}<0.001)$ as well as PFS (5.4 months vs 1.4 months, $\mathrm{P}<0.001)$ and OS $(9.6$ months vs 6.3 months, $\mathrm{P}=0.002)^{6}$

Relatively few real-world studies have reported the use of anlotinib in advanced NSCLC patients. Thus, here, we retrospectively studied the efficacy and safety of anlotinib as third- or further-line treatment against advanced NSCLC. The ORR and DCR were $16 \%$ and $80 \%$, respectively. In addition, the mPFS and mOS were 4.5 and 9 months, respectively, consistent with the results of the ALTER0303 trial. These findings indicate that anlotinib treatment is effective in patients with advanced NSCLC in real-world settings. A previous retrospective study only focused on the PFS of anlotinib. ${ }^{14}$ To the best of our knowledge this is the first real-world study evaluating the 
Table 2 Univariate Analysis of Progression-Free Survival (PFS)

\begin{tabular}{|c|c|c|c|}
\hline Group & mPFS & $95 \% \mathrm{Cl}$ & $\mathbf{P}$ \\
\hline Sex & & & 0.915 \\
\hline Male & 5 & $3.7-6.3$ & \\
\hline Female & 4.5 & $3.5-5.5$ & \\
\hline Age & & & 0.336 \\
\hline$<65$ & 4.5 & $2.5-6.5$ & \\
\hline $65-75$ & 4 & $2.5-5.5$ & \\
\hline$\geq 75$ & 5 & $3.5-6.5$ & \\
\hline Smoking history & & & 0.672 \\
\hline Yes & 5 & $3.8-6.2$ & \\
\hline No & 4.5 & $2.8-6.2$ & \\
\hline ECOG PS & & & 0.000 \\
\hline$\leq 1$ & 5 & $4.4-5.6$ & \\
\hline 2 & 2.5 & $1.0-4.1$ & \\
\hline Pathological type & & & 0.292 \\
\hline Adenocarcinoma & 4 & $3.2-4.8$ & \\
\hline Squamous cell carcinoma & 5.3 & $4.9-5.7$ & \\
\hline Gene status & & & 0.941 \\
\hline EGFR mutation & 4.5 & $3.5-5.5$ & \\
\hline Wide type/unknown & 5 & $3.8-6.2$ & \\
\hline Clinical stage & & & 0.389 \\
\hline III B & 5 & $1.1-8.9$ & \\
\hline IV & 4.5 & $3.5-5.5$ & \\
\hline Number of distant metastases & & & 0.009 \\
\hline$\leq 2$ & 5 & $4.4-5.6$ & \\
\hline$>2$ & 3.5 & $2.9-4.1$ & \\
\hline Brain metastases & & & 0.237 \\
\hline Yes & 4 & $3-5$ & \\
\hline No & 5 & $4.3-5.7$ & \\
\hline Liver metastases & & & 0.000 \\
\hline Yes & 2 & $0-4$ & \\
\hline No & 5 & $4.4-5.6$ & \\
\hline Number of previous treatment lines & & & 0.012 \\
\hline$\leq 3$ & 5 & $4.4-5.6$ & \\
\hline$>3$ & 2.5 & $1.7-3.3$ & \\
\hline Number of previous chemotherapy lines & & & 0.029 \\
\hline$\leq 2$ & 5 & $4.4-5.6$ & \\
\hline$>2$ & 2.8 & $1.1-4.5$ & \\
\hline Previous EGFR-TKI treatment & & & 0.763 \\
\hline Yes & 4 & $2.9-5.1$ & \\
\hline No & 5 & $4-6$ & \\
\hline Previous antiangiogenic treatment & & & 0.276 \\
\hline Yes & 4 & $2.5-5.5$ & \\
\hline No & 5 & $4.2-5.8$ & \\
\hline
\end{tabular}

Abbreviations: $\mathrm{mPFS}$, median progression-free survival; $\mathrm{Cl}$, confidence interval; ECOG PS, Eastern Cooperative Oncology Group performance status; EGFR, endothelial growth factor receptor; TKI, tyrosine kinase inhibitor.
Table 3 Cox Regression Analysis of Progression-Free Survival (PFS)

\begin{tabular}{|l|l|l|l|}
\hline Group & $\mathbf{P}$ & $\mathbf{H R}$ & $\mathbf{9 5 \%} \mathbf{~ C I}$ \\
\hline $\begin{array}{c}\text { ECOG PS } \\
\leq I \text { vs 2 }\end{array}$ & 0.003 & 0.308 & $0.14 \mathrm{I}-0.673$ \\
\hline $\begin{array}{c}\text { Liver metastases } \\
\text { No vs yes }\end{array}$ & 0.000 & 0.197 & $0.079-0.489$ \\
\hline
\end{tabular}

Abbreviations: HR, hazard ratio; $\mathrm{Cl}$, confidence interval; ECOG PS, Eastern Cooperative Oncology Group performance status.

OS of anlotinib as third or further-line treatment in patients with advanced NSCLC.

Among the 50 patients who received anlotinib over more than one cycle, 20 aged $\geq 65$ years, of which 10 aged $>75$ years. Subgroup analyses showed that $\mathrm{mPFS}$ and $\mathrm{mOS}$ did not differ among patients aged $<65,65-75$, and $\geq 75$ years. The mOS of patients aged $<65,65-75$, and $\geq 75$ years was 8 months (95\% CI: 4.5-11.5), 8 months (95\% CI: 3.9-12.1), and 9 months (95\% CI: 5.1-12.9), respectively. In the ALTER0303 trial, the mOS of patients aged 70-75 years receiving anlotinib as third-line or further treatment was 14.5 months; in contrast, the mOS of patients aged 60-70 years and those aged $<60$ years was 7.9 and 10.1 months, respectively. ${ }^{6}$ These results suggest that elderly patients, especially those aged $>75$ years, may benefit from anlotinib treatment.

Previous studies have shown that antiangiogenic drugs are more effective in patients with EGFR-sensitive mutations. ${ }^{12,15}$ In our study, there were no differences in the MPFS and mOS of patients with EGFR mutations or wild-type/unknown EGFR status, similar to the results of the ALTER0303 study, indicating that anlotinib is effective in advanced NSCLC regardless of the EGFR status. Although the incidence of EGFR mutations in Caucasian and Asian populations with NSCLC is $10-20 \%$ and $40-60 \%$, respectively, ${ }^{16,17}$ anlotinib may still be effective in Caucasian patients with NSCLC; however, this should be investigated in a clinical trial. Moreover, the mOS of patients with no driver gene mutation who received anlotinib as a third-line or further treatment was 9.5 months in this trial, whereas the OS of patients who received docetaxel alone or in combination with ramucirumab as a second-line treatment is 9.5 and 10.5 months, respectively. ${ }^{10}$ Therefore, a study investigating the use of anlotinib combined with docetaxel as second-line treatment in patients with advanced NSCLC would be worthwhile.

Previous studies have demonstrated that ECOG PS is an important predictor of prognosis in patients with 



Figure 2 Overall survival of patients with advanced non-small lung cancer treated with anlotinib. (A) total population $(n=50)$, (B) Eastern Cooperative Oncology Group performance status (ECOG PS), (C) number of distant metastases, (D) liver metastases, (E) number of previous treatment lines, (F) number of previous chemotherapy lines.

advanced NSCLC. ${ }^{18,19}$ In our study, Cox regression analysis showed that patients with ECOG PS $\leq 1$ treated with anlotinib had longer PFS (5 months vs 2.5 months) and OS (9.5 months vs 5 months) than patients with ECOG PS 2. In the ALTER0303 trial, the mOS of patients with ECOG PS 0 was 15.1 months, whereas that of patients with ECOG PS 1 was 8.8 months. These results indicate that anlotinib is more effective in patients with ECOG PS $\leq 1$.

In our study, the mPFS and mOS of anlotinib in patients without liver metastasis was 4 and 9.5 months, whereas the mPFS and mOS of patients with liver metastases was 2 and 5 months, respectively, showing that anlotinib alone had limited efficacy in patients with advanced NSCLC and liver metastases. This finding is similar to that of a previous study, in which the PFS anlotinib in NSCLC patients with and without liver metastases was 3 months and 5 months, respectively. ${ }^{14}$ Moreover, the ALTER0303 trial did not report anlotinib efficacy in patients with liver metastases. In our study, among the 8 patients with liver metastasis, 5 patients had $>2$ distant metastases and 6 patients had $>3$ previous treatment lines, which could have limited the efficacy of anlotinib. Multiple studies have shown that the presence of liver metastases is a poor prognostic factor in patients with NSCLC. ${ }^{18,20,21}$ However, the IMpower150 study showed that atezolizumab, an immune-checkpoint inhibitor, combined with chemotherapy plus bevacizumab improved OS in advanced NSCLC patients with liver metastasis compared with chemotherapy plus bevacizumab. ${ }^{22}$ The efficacy of anlotinib combined with immune-checkpoint inhibitors and chemotherapy in patients with advanced NSCLC needs to be explored in the future.

Bleeding is a serious adverse event associated with antiangiogenic drugs, and previous studies have shown that sunitinib, sorafenib, and bevacizumab can cause fatal pulmonary hemorrhage. ${ }^{23}$ In this study, the incidence of hemoptysis was $10 \%$, and only one patient with adenocarcinoma 
Table 4 Univariate Analysis of Overall Survival (OS)

\begin{tabular}{|c|c|c|c|}
\hline Group & mos & $95 \% \mathrm{Cl}$ & $\mathbf{P}$ \\
\hline Sex & & & 0.372 \\
\hline Male & 8 & $4.9-11.1$ & \\
\hline Female & 9.3 & $6.4-12.2$ & \\
\hline Age & & & 0.964 \\
\hline$<65$ & 8 & $4.5-11.5$ & \\
\hline $65-75$ & 8 & $3.9-12.1$ & \\
\hline$\geq 75$ & 9 & $5.1-12.9$ & \\
\hline Smoking history & & & 0.591 \\
\hline Yes & 8 & $4.6-11.4$ & \\
\hline No & 9.3 & $7.3-11.3$ & \\
\hline ECOG PS & & & 0.000 \\
\hline$\leq 1$ & 9.5 & $8.6-10.4$ & \\
\hline 2 & 5 & $3.5-6.5$ & \\
\hline Pathological type & & & 0.229 \\
\hline Adenocarcinoma & 7 & $2.6-11.4$ & \\
\hline Squamous cell carcinoma & 10 & $4.9-5.7$ & \\
\hline Gene status & & & 0.714 \\
\hline EGFR mutation & 8 & $4.3-11.7$ & \\
\hline Wide type/unknown & 9.5 & $7.1-11.9$ & \\
\hline Clinical stage & & & 0.14 \\
\hline III B & 10 & $7-13$ & \\
\hline IV & 8 & $4.3-11.7$ & \\
\hline Number of distant metastases & & & 0.004 \\
\hline$\leq 2$ & 9.5 & $8.7-10.3$ & \\
\hline$>2$ & 5 & $3.2-6.8$ & \\
\hline Brain metastases & & & 0.265 \\
\hline Yes & 7 & $0.8-13.2$ & \\
\hline No & 9 & $6.2-11.8$ & \\
\hline Liver metastases & & & 0.000 \\
\hline Yes & 4 & $2.7-5.3$ & \\
\hline No & 9.5 & $8.6-10.4$ & \\
\hline Number of previous treatments & & & 0.002 \\
\hline$\leq 3$ & 9.5 & $8.8-10.2$ & \\
\hline$>3$ & 5.5 & $4.5-6.5$ & \\
\hline Number of previous chemotherapy lines & & & 0.008 \\
\hline$\leq 2$ & 9.5 & $8.3-10.7$ & \\
\hline$>2$ & 6 & $2.6-9.4$ & \\
\hline Previous EGFR-TKI treatment & & & 0.822 \\
\hline Yes & 7 & $3.1-10.9$ & \\
\hline No & 9.5 & $7.4-11.6$ & \\
\hline Previous antiangiogenic treatment & & & 0.158 \\
\hline Yes & 8 & $4.8-11.2$ & \\
\hline No & 9.3 & $5.9-12.7$ & \\
\hline
\end{tabular}

Abbreviations: $\mathrm{Cl}$, confidence interval; ECOG PS, Eastern Cooperative Oncology Group performance status; EGFR, endothelial growth factor receptor; TKI, tyrosine kinase inhibitor.
Table 5 Cox Regression Analysis of Overall Survival (OS)

\begin{tabular}{|l|l|l|l|}
\hline Group & $\mathbf{P}$ & $\mathbf{H R}$ & $\mathbf{9 5 \%} \mathbf{~ C l}$ \\
\hline $\begin{array}{c}\text { ECOG PS } \\
\leq \text { I vs 2 }\end{array}$ & 0.004 & 0.302 & $0.091-0.684$ \\
\hline $\begin{array}{c}\text { Liver metastases } \\
\text { No vs yes }\end{array}$ & 0.000 & 0.065 & $0.004-0.214$ \\
\hline
\end{tabular}

Abbreviations: $\mathrm{HR}$, hazard ratio; $\mathrm{Cl}$, confidence interval; ECOG PS, Eastern Cooperative Oncology Group performance status.

Table 6 Treatment-Related Adverse Events

\begin{tabular}{|l|l|l|}
\hline & N = 52 \\
\hline & All grades & Grade 3 \\
Hypertension & I3 (25\%) & $4(8 \%)$ \\
Fatigue & II (2I\%) & I (2\%) \\
Hand-foot syndrome & I0 (2I\%) & 0 \\
Proteinuria & $9(17 \%)$ & I (2\%) \\
Anorexia & $8(15 \%)$ & 0 \\
Constipation & $7(13 \%)$ & 0 \\
Diarrhea & $7(13 \%)$ & 0 \\
Oral mucositis & $6(12 \%)$ & 0 \\
Abnormal liver function & $6(12 \%)$ & 0 \\
Cough & $5(10 \%)$ & $1(2 \%)$ \\
Hemoptysis & $5(10 \%)$ & $1(2 \%)$ \\
Rash & $4(8 \%)$ & 0 \\
Thrombocytopenia & $3(6 \%)$ & 0 \\
\hline
\end{tabular}

developed grade 3 hemoptysis. Hypertension is a common adverse reaction to antiangiogenic drugs and can be controlled by using antihypertensive drugs. The incidence of hypertension in this study was $25 \%$; only four patients (8\%) reported grade 3 hypertension. Other common adverse events included fatigue, hand-foot syndrome, proteinuria, and anorexia, which were similar to those reported in previous studies of anlotinib. ${ }^{24-26}$ Moreover, only two patients discontinued anlotinib treatment due to grade 3 hypertension or hemoptysis, and only five received dose reductions. These findings indicate that anlotinib is well tolerated in NSCLC.

Notable limitations of this study are that our data were obtained from a single medical center with a small number of patients; thus, larger observational studies should be conducted in future to validate these results. In addition, we were unable to investigate the presence of predictive biomarkers for anlotinib efficacy owing to the unavailability of tumor tissue or plasma samples before treatment. Previously, efforts have been made to identify predictive biomarkers for antiangiogenic drugs in patients with advanced NSCLC. Tumor tissue biomarkers include microvascular density ${ }^{27}$ and 
VEGFR2, ${ }^{28}$ whereas plasma biomarkers include VEGFA ${ }^{29,30}$ and circulating endothelial cells. ${ }^{31}$ Moreover, CD31-labeled circulating endothelial cells were used to predict the efficacy of anlotinib in the ALTER0303 study. ${ }^{32}$ Future studies should aim to identify the best predictive biomarkers to evaluate the efficacy of anlotinib in the treatment of advanced NSCLC.

\section{Conclusion}

Our retrospective study revealed that anlotinib was well tolerated and effective in patients with advanced NSCLC in a real-world clinical setting. Patients with an ECOG PS $\leq 1$ and no liver metastasis showed longer PFS and OS.

\section{Funding}

This work was supported by the Chinese National Instrumentation Program, No. 2011YQ170067, and Beijing Municipal Science \& Technology Commission, PR China (No. Z181100001718074).

\section{Disclosure}

The authors report no conflicts of interest for this study.

\section{References}

1. Bray F, Ferlay J, Soerjomataram I, Siegel RL, Torre LA, Jemal A. Global cancer statistics 2018: GLOBOCAN estimates of incidence and mortality worldwide for 36 cancers in 185 countries. CA Cancer J Clin. 2018;68(6):394-424. doi:10.3322/caac.21492

2. Molina JR, Yang P, Cassivi SD, Schild SE, Adjei AA. Non-small cell lung cancer: epidemiology, risk factors, treatment, and survivorship. Mayo Clin Proc. 2008;83(5):584-594. doi:10.1016/S0025-6196(11) 60735-0

3. Arbour KC, Riely GJ. Systemic therapy for locally advanced and metastatic non-small cell lung cancer: a review. JAMA. 2019;322 (8):764-774. doi:10.1001/jama.2019.11058

4. Ferrara N, Adamis AP. Ten years of anti-vascular endothelial growth factor therapy. Nat Rev Drug Discov. 2016;15(6):385-403. doi: $10.1038 / \mathrm{nrd} .2015 .17$

5. Xie C, Wan X, Quan H, et al. Preclinical characterization of anlotinib, a highly potent and selective vascular endothelial growth factor receptor-2 inhibitor. Cancer Sci. 2018;109(4):1207-1219. doi:10.1111/cas. 13536

6. Han B, Li K, Wang Q, et al. Effect of anlotinib as a third-line or further treatment on overall survival of patients with advanced non-small cell lung cancer: the ALTER 0303 Phase 3 randomized clinical trial. JAMA Oncol. 2018;4(11):1569-1575. doi:10.1001/ jamaoncol.2018.3039

7. Nabhan C, Klink A, Prasad V. Real-world evidence-what does it really mean? JAMA Oncol. 2019;5(6):781-783. doi:10.1001/jamaoncol. 2019.0450

8. Reck M, von Pawel J, Zatloukal P, et al. Phase III trial of cisplatin plus gemcitabine with either placebo or bevacizumab as first-line therapy for nonsquamous non-small-cell lung cancer: AVAil. J Clin Oncol. 2009;27(8):1227-1234. doi:10.1200/JCO.2007.14.5466

9. Sandler A, Gray R, Perry MC, et al. Paclitaxel-carboplatin alone or with bevacizumab for non-small-cell lung cancer. $N$ Engl $J$ Med. 2006;355(24):2542-2550. doi:10.1056/NEJMoa061884
10. Zhou C, Wu YL, Chen G, et al. BEYOND: a randomized, double-blind, placebo-controlled, multicenter, phase III study of first-line carboplatin/paclitaxel plus bevacizumab or placebo in Chinese patients with advanced or recurrent nonsquamous non-smallcell lung cancer. J Clin Oncol. 2015;33(19):2197-2204. doi:10.1200/ JCO.2014.59.4424

11. Garon EB, Ciuleanu T-E, Arrieta O, et al. Ramucirumab plus docetaxel versus placebo plus docetaxel for second-line treatment of stage IV non-small-cell lung cancer after disease progression on platinum-based therapy (REVEL): a multicentre, double-blind, randomised phase 3 trial. Lancet. 2014;384(9944):665-673. doi:10.1016/S0140-6736(14)60845-X

12. Saito H, Fukuhara T, Furuya N, et al. Erlotinib plus bevacizumab versus erlotinib alone in patients with EGFR-positive advanced non-squamous non-small-cell lung cancer (NEJ026): interim analysis of an open-label, randomised, multicentre, phase 3 trial. Lancet Oncol. 2019;20(5):625-635. doi:10.1016/S1470-2045(19)30035-X

13. Lu S, Chang J, Liu X, et al. Randomized, double-blind, placebo-controlled, multicenter Phase II study of fruquintinib after two prior chemotherapy regimens in Chinese patients with advanced nonsquamous non small-cell lung cancer. J Clin Oncol. 2018;36 (12):1207-1217. doi:10.1200/JCO.2017.76.7145

14. Shao L, Wang W, Song Z, Zhang Y. The efficacy and safety of anlotinib treatment for advanced lung cancer. Onco Targets Ther. 2019;12:6549-6554. doi:10.2147/OTT.S205674

15. Paz-Ares L, Hirsh V, Zhang L, et al. Monotherapy administration of sorafenib in patients With non-small cell lung cancer (MISSION) Trial: a Phase III, multicenter, placebo-controlled trial of sorafenib in patients with relapsed or refractory predominantly nonsquamous non-small-cell lung cancer after 2 or 3 previous treatment regimens. $J$ Thorac Oncol. 2015;10(12):1745-1753. doi:10.1097/JTO.0 000000000000693

16. Hsu WH, Yang JC, Mok TS, Loong HH. Overview of current systemic management of EGFR-mutant NSCLC. Ann Oncol. 2018;29(suppl_1):i3-i9. doi:10.1093/annonc/mdx702

17. Midha A, Dearden S, McCormack R. EGFR mutation incidence in non-small-cell lung cancer of adenocarcinoma histology: a systematic review and global map by ethnicity (mutMapII). Am J Cancer Res. 2015;5(9):2892-2911.

18. Castanon E, Rolfo C, Vinal D, et al. Impact of epidermal growth factor receptor (EGFR) activating mutations and their targeted treatment in the prognosis of stage IV non-small cell lung cancer (NSCLC) patients harboring liver metastasis. $J$ Transl Med. 2015;13:257. doi:10.1186/s12967-015-0622-x

19. Sculier JP, Chansky K, Crowley JJ, Van Meerbeeck J, Goldstraw P, International Staging Committee and Participating Institutions. The impact of additional prognostic factors on survival and their relationship with the anatomical extent of disease expressed by the 6th edition of the TNM classification of malignant tumors and the proposals for the 7th edition. $J$ Thorac Oncol. 2008;3(5):457-466. doi:10.1097/JTO.0b013e31816de2b8

20. Riihimaki M, Hemminki A, Fallah M, et al. Metastatic sites and survival in lung cancer. Lung Cancer. 2014;86(1):78-84. doi:10.1016/j.lungcan.2014.07.020

21. Tumeh PC, Hellmann MD, Hamid O, et al. Liver metastasis and treatment outcome with anti-PD-1 monoclonal antibody in patients with melanoma and NSCLC. Cancer Immunol Res. 2017;5 (5):417-424. doi:10.1158/2326-6066.CIR-16-0325

22. Reck M, Mok TSK, Nishio M, et al. Atezolizumab plus bevacizumab and chemotherapy in non-small-cell lung cancer (IMpower150): key subgroup analyses of patients with EGFR mutations or baseline liver metastases in a randomised, open-label phase 3 trial. Lancet Respir Med. 2019;7(5):387-401. doi:10.1016/S2213-2600(19)30084-0

23. Piperdi B, Merla A, Perez-Soler R. Targeting angiogenesis in squamous non-small cell lung cancer. Drugs. 2014;74(4):403-413. doi:10.1007/s40265-014-0182-z 
24. Han B, Li K, Zhao Y, et al. Anlotinib as a third-line therapy in patients with refractory advanced non-small-cell lung cancer: a multicentre, randomised phase II trial (ALTER0302). $\mathrm{Br}$ $J$ Cancer. 2018;118(5):654-661. doi:10.1038/bjc.2017.478

25. Si X, Zhang L, Wang $\mathrm{H}$, et al. Management of anlotinib-related adverse events in patients with advanced non-small cell lung cancer: experiences in ALTER-0303. Thorac Cancer. 2019;10(3):551-556. doi:10.1111/1759-7714.12977

26. Wu D, Nie J, Dai L, et al. Salvage treatment with anlotinib for advanced non-small cell lung cancer. Thorac Cancer. 2019;10 (6):1590-1596. doi:10.1111/1759-7714.13120

27. Zhao YY, Xue C, Jiang W, et al. Predictive value of intratumoral microvascular density in patients with advanced non-small cell lung cancer receiving chemotherapy plus bevacizumab. J Thorac Oncol. 2012;7(1):71-75. doi:10.1097/JTO.0b013e31823085f4

28. Kim ES, Herbst RS, Wistuba II, et al. The BATTLE trial: personalizing therapy for lung cancer. Cancer Discov. 2011;1(1):44-53. doi:10.1158/2159-8274.CD-10-0010
29. Hanrahan EO, Ryan AJ, Mann H, et al. Baseline vascular endothelial growth factor concentration as a potential predictive marker of benefit from vandetanib in non-small cell lung cancer. Clin Cancer Res. 2009;15(10):3600-3609. doi:10.1158/1078-0432.CCR-08-2568

30. Hegde PS, Jubb AM, Chen D, et al. Predictive impact of circulating vascular endothelial growth factor in four phase III trials evaluating bevacizumab. Clin Cancer Res. 2013;19(4):929-937. doi:10.1158/ 1078-0432.CCR-12-2535

31. Reinmuth N, Thomas M, Meister M, Schnabel PA, Kreuter M. Current data on predictive markers for anti-angiogenic therapy in thoracic tumours. Eur Respir J. 2010;36(4):915-924. doi:10.1183/ 09031936.00074009

32. Liu Z, Wang J, Meng Z, et al. CD31-labeled circulating endothelial cells as predictor in anlotinib-treated non-small-cell lung cancer: analysis on ALTER-0303 study. Cancer Med. 2018;7(7):3011-3021. doi:10.1002/cam4.1584

\section{Publish your work in this journal}

Cancer Management and Research is an international, peer-reviewed open access journal focusing on cancer research and the optimal use of preventative and integrated treatment interventions to achieve improved outcomes, enhanced survival and quality of life for the cancer patient.
The manuscript management system is completely online and includes a very quick and fair peer-review system, which is all easy to use. Visit http://www.dovepress.com/testimonials.php to read real quotes from published authors. 